\title{
Intraventricular Tension Pneumocephalus and Pneumorrhachis in a Dog; Diagnosis, Treatment, and MRI Follow-Up
}

\author{
X Raurell ${ }^{1 *}$, C Centellas ${ }^{1}$, G Feliz ${ }^{1}$, Francisca Almeida ${ }^{1}$, A Zamora ${ }^{2}$ and \\ M Pumarola $^{3}$ \\ ${ }^{1}$ Neurology and Neurosurgery, Hospital Veterinari Molins, Barcelona, Spain \\ ${ }^{2}$ Diagnostic Imaging, Hospital Veterinari Molins, Barcelona, Spain \\ ${ }^{3}$ Unitat de Patologia Murina i Comparada, Veterinary Faculty of Autonomous University \\ of Barcelona, Spain \\ *Corresponding Author: X Raurell, Neurology and Neurosurgery, Hospital Veterinari \\ Molins, Barcelona, Spain.
}

DOI: $10.31080 /$ ASVS.2020.02.0052

\section{Abstract}

Pneumocephalus and pneumorrhachis are the accumulation of air within the cranial cavity and vertebral canal respectively being uncommon and life-threatening complications after craniotomies and few reports have been published in the veterinary literature.

An 8-year-old crossbreed male dog was presented due to the development of two generalized tonic-clonic seizures during the same night. The MRI of the brain showed a right frontal-olfactory mass compatible with a meningioma which was confirmed by pathology. Eight days after the transfrontal craniotomy the dog presented neurological signs that progressed to a decerebrate posture and mydriasis in 24h. An intraventricular pneumocephalus and subarachnoid pneumorrhachis were detected in a second MRI. Immediate reoperation was carried out. At 8 weeks after reoperation, a third MRI was performed in which a complete resolution of the pneumocephalus was observed. After ten months without showing any neurological sign, this patient was euthanized due to status epilepticus by the referral veterinarian.

Close monitoring, early identification of postsurgical clinical signs and imaging features are critical in order to treat this condition successfully and good long term prognosis may be achieved.

Keywords: Pneumocephalus; Pneumorrhachis; Magnetic Resonance Imaging; Craniotomy; Meningioma; Dog

\section{Abbreviations}

MRI: Magnetic Resonance Imaging; T1W: T1 Weigthed Image; T2W: T2 Weigthed Image; FLAIR: Fluid-Attenuated Inversion Recovery; T2*: Gradient-Echo Sequence; CSF: Cerebrospinal Fluid; C1: First Cervical Vertebra; C2: Second Cervical Vertebra.

\section{Introduction}

Pneumocephalus is the accumulation of air within the cranial cavity [1]. Pneumorrhachis is the accumulation of air inside the vertebral canal [2]. It can be asymptomatic or under tension when the accumulation of air induces neurological signs that might be lethal [3]. The air accumulation can occur in the epidural, subdural, subarachnoid spaces, it can be intraventricular or intraparenchymal [3]. It may be seen as a complication after a craniotomy [2-6] and trauma [7-9] but other causes are reported in the veterinary literature $[10,11]$. In this case report, we present an 8-year-old crossbreed male dog that developed an intraventricular tension pneumocephalus and pneumorrhachis as a complication of a transfrontal craniotomy performed to treat a meningioma.

\section{Case Details}

An 8-year-old crossbreed sexually intact male dog was admitted due to the development of two generalized tonic-clonic seizures on the same night. The patient was already diagnosed and treated for hypothyroidism, dewormed with fluralaner in the previous day and did not have access to toxic substances, although it lived in a house with a garden.

The patient's physical exam was normal and obnubilation was observed as a neurological sign. A diagnostic approach that consisted of complete blood count, biochemistry profile, urine analysis, chest radiographs in two orthogonal projections, abdominal ultrasound and magnetic resonance of the cranium was performed. For the MRI study, a low-field magnet was used (MRgrande 0,25T, 
Esaote, Genoa, Italy) and T1W, T2W, and postcontrast T1W sequences were obtained in all different planes of space, while FLAIR and $\mathrm{T}^{*}$ sequences were obtained only in transverse sections. A well-defined mass was seen in the right olfactory region. Perilesional vasogenic edema associated with the mass was observed, and together they exerted a mass effect with midline shift, ventricular distortion, the collapse of the sulci and diencephalic distortion as well. The mass presented a heterogeneous signal with a hemorrhagic and cystic intratumoral component with marked contrast enhancement. This study was compatible with a right frontal-olfactory meningioma, histiocytic sarcoma, metastasic neoplasia and lymphoma (Figure 1).

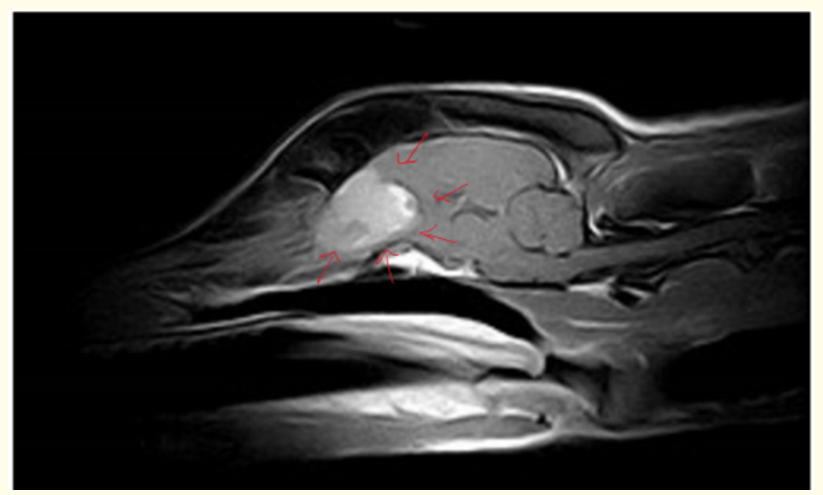

Figure 1: Right parasagittal T1-weighted contrast-enhanced image. Well-defined frontal-olfactory mass with marked contrast enhancement. The intratumoral hypodense area was thought to represent necrosis.

The patient was administered with phenobarbital $(2.5 \mathrm{mg} / \mathrm{kg}$ twice daily) and prednisone $(0.5 \mathrm{mg} / \mathrm{kg} /$ twice daily) therapy and surgical treatment was proposed. A transfrontal craniotomy was performed four days after the MRI. The histopathologic diagnosis was a meningothelial meningioma (Figure 2).

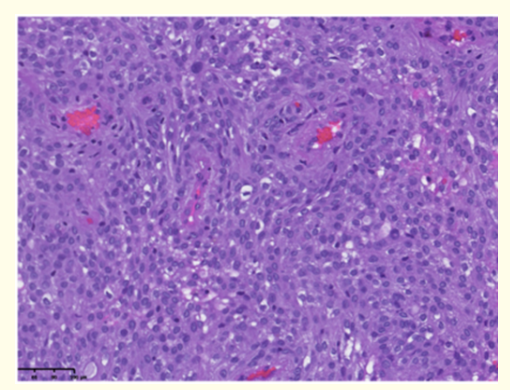

Figure 2: This meningioma consisted of cells with ovoid nuclei, prominent nucleolus and scarce citoplasm. It had a low mitotic index. Although the meningothelial pattern predominated, it also

had microcystic and fibrous patterns. H/E staining x 40 .
Eight days after the surgery the patient presented right unilateral haemorrhagic nasal discharge and neurological signs that progressed in 24 hours from obnubilation, miosis, head tilt to the right and proprioceptive deficits in the four limbs to a decerebration posture and mydriasis. This was treated as a matter of emergency with hypertonic saline oxygen therapy and a second MRI was performed using the same protocol as previously mentioned. It showed an intraventricular signal void in all sequences suggestive of air, lateral ventricular distension with a certain degree of asymmetry giving the ventricles a more rounded shape, a meniscus shape between de CSF and the air inside the left lateral ventricle (Figures 3A, 3B), distension of the III and IV ventricles and mesencephalic aqueduct and the existence of subarachnoid air dorsally in the spinal cord, between C1 and C2 (Figure 4). An air-filled fistula was not observed. Besides these findings, postsurgical changes, tumoral remaining in the olfactory region and existence of air in the frontal right epidural space with the slight distortion of the frontal lobe were observed.

\section{A}

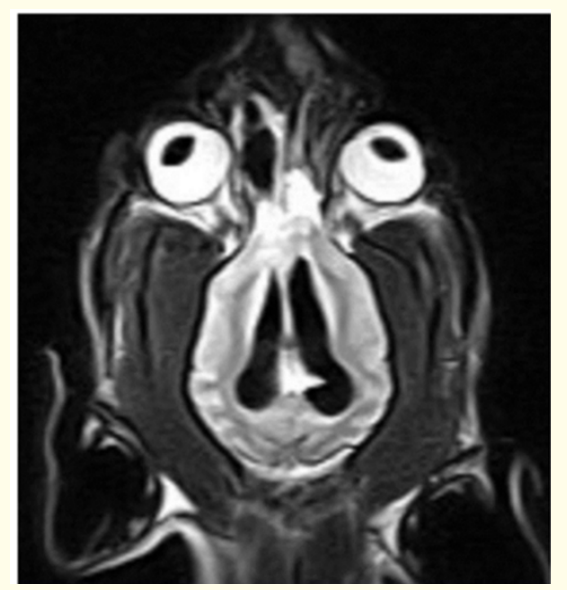

B

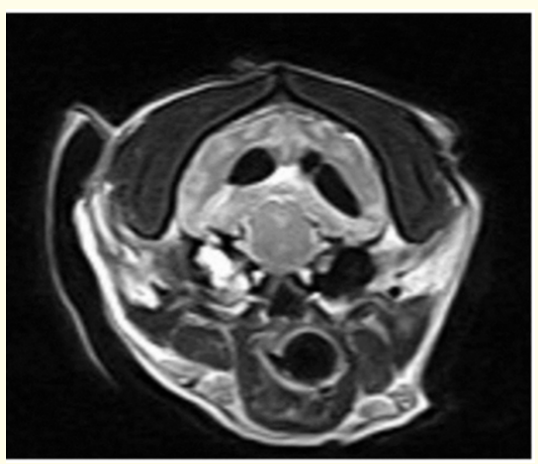

Figure 3: A) Dorsal T2-weighted image at the level of cerebral hemipheres. Intraventricular signal void and distension of the lateral ventricles can be seen. B) Transverse T2-weighted image at the level of midbrain. Meniscus shape between CSF and signal void. The latter is compatible with air. The right tympanic bulla is filled with an hyperintense material which was considered an incidental finding. 


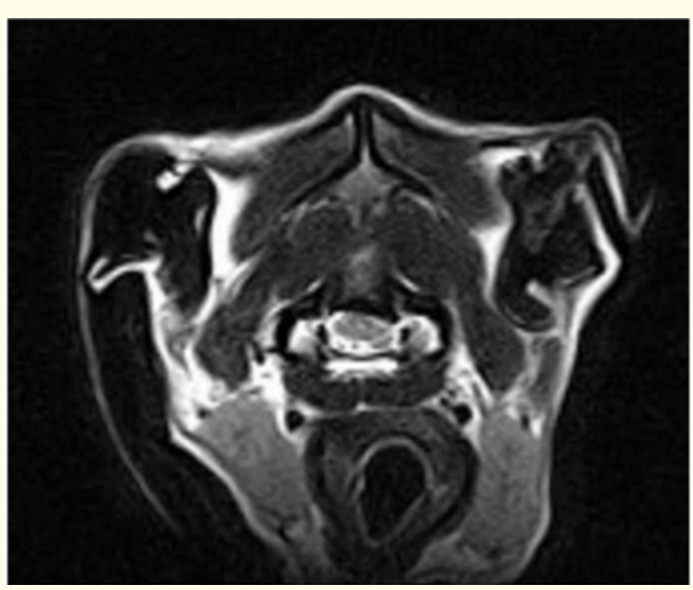

Figure 4: Transverse T2-weighted image at the level of the atlas. Subarachnoid air is seen dorsally to the spinal cord.

Immediate revision surgery was carried out. Washings were performed with physiological saline, residues of grey and amorphous substance were removed at the right olfactory level and the dural defect was closed by placing a cellulose patch and a graft of fascia from temporal muscle on the exposed brain parenchyma imitating the recoating of the dura mater. Furthermore, the opening in the inner frontal bone was sealed with bone cement. The patient maintained consciousness, presented cranial reflexes as well as the menace response, responded to stimuli and was able to eat $24 \mathrm{~h}$ after the revision surgery. The patient was discharged 6 days after the second surgery with phenobarbital $(2,5 \mathrm{mg} / \mathrm{kg}$ twice daily), prednisone $(0,5 \mathrm{mg} / \mathrm{kg}$ twice daily) and trimethoprim sulfa (30 $\mathrm{mg} / \mathrm{kg}$ twice daily) therapy. Toceranib chemotherapy was started three times per week at the 10 days check up.

At 8 weeks after reintervention, a third MRI was carried out in which a complete resolution of the pneumocephalus was observed at the frontal, ventricular and subarachnoid levels and there were no signs of tumor regrowth (Figure 5). Thus prednisone was slowly decreased. At 3, 4, 5 and 6 months examinations after the second surgery, the neurological examination was still normal and the patient did not have additional seizures. After ten months without showing any neurologic sign, this patient died due to status epilepticus at the referral veterinarian.

\section{Results and Discussion}

The accumulation of air inside the cranium is a very common phenomenon in people after trauma or craniotomies and it is usually asymptomatic $[1,3]$. Tension pneumocephalus (symptomatic pneumocephalus) occurs when air accumulates under pressure producing clinical signs that are usually progressive and of diffuse localization $[1-3,11]$. In veterinary medicine, there are nine

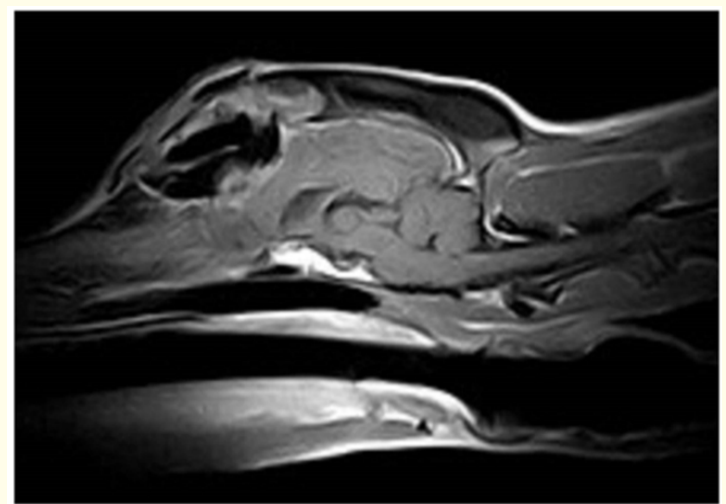

Figure 5: Sagittal post-contrast T1-weighted image. Resolution of pneumocephalus and no signs of neoplastic regrowth are shown. Post-surgical changes are seen in the frontal sinus.

sporadic cases described and it is considered a rare condition [25,7-11]. Pneumocephalus due to a complication after transfrontal craniotomy has been reported only in 4 cases previously [2-5] and pneumorrhachis only in one [2].

Tension pneumocephalus originates when there is a lack of continuity through the cranium and meninges and by the establishment of a pressure gradient between atmospheric and intracranial pressures [2-4].

In this case report, the factors predisposing to pneumocephalus could have been the lack of continuity created by surgery, the unilateral hemorrhagic nasal discharge between surgery and the presentation of signs, the probable leakage of CSF with epistaxis, the removal of a frontal-olfactory mass of considerable dimensions and the non-repair of the dural defect in the first surgery.

\section{Conclusion}

In conclusion, this case suggests that if tension pneumocephalus is detected and treated immediately, it may have a favorable long term prognosis. Tension pneumocephalus should be considered as a potential complication in patients showing deteriorating neurological signs after craniotomy being those patients candidates for repeat imaging.

\section{Conflict of Interest}

Authors declare that no financial interest or conflict of interest exists.

\section{Bibliography}

1. Osborn AG. "Pneumocephalus (skull, scalp, and meninges)". Diagnostic Imaging Brain. Amirsys II-4 (2004): 18-21.

2. Cavanaugh RP., et al. "Intraventricular Tension Pneumocepha- 
lus and Cervical Subarachnoid Pneumorrhachis In a Bull Mastiff Dog After Craniotomy". Journal of Small Animal Practice 49.5 (2008): 244-248.

3. Garosi LS., et al. "Intraventricular tension pneumocephalus as a complication of transfrontal craniectomy: a case report". Veterinary Surgery 31 (2002): 226-231.

4. Garosi LS., et al. "What is your diagnosis? Pneumocephalus". Journal of the American Veterinary Medical Association 226.7 (2005): 1057-1058.

5. Ros C., et al. "What is your diagnosis? Pneumocephalus". Journal of the American Veterinary Medical Association 246.9 (2015): 959-961.

6. Uriarte A and Capello R. Transfrontal craniotomy. Current Techniques in Canine and Feline Neurosurgery (Edited by Andy Shores and Bigitte A. Brisson). Wiley Blackwell (2017): 99-107.

7. Hecht S. Brain trauma-Pneumocephalus. "Diagnostic MRI in Dogs and Cats (Edited by Wilfried Mai)". CRC Press (2018): 314.

8. Haley AC and Abramson C. "Traumatic pneumocephalus in a dog". Journal of the American Veterinary Medical Association 234.10 (2009): 1295-1298.

9. Thieman K., et al. "Imaging diagnosis: trauma-induced tension pneumocephalus". Veterinary Radiology and Ultrasound 49 (2008): 362-364.

10. Fletcher D., et al. "Ventricular pneumocephalus and septic meningoencephalitis secondary to dorsal rhinotomy and nasal polypectomy in a dog". Journal of the American Veterinary Medical Association 229.6 (2006): 240-245.

11. Shea A., et al. "Spontaneous non-traumatic tension pneumocephalus in a dog with nasal meningoencephalocele". Veterinary Record 6.4 (2018): 1-4.

\section{Assets from publication with us}

- Prompt Acknowledgement after receiving the article

- Thorough Double blinded peer review

- Rapid Publication

- Issue of Publication Certificate

- High visibility of your Published work

Website: www.actascientific.com/

Submit Article: www.actascientific.com/submission.php

Email us: editor@actascientific.com

Contact us: +919182824667

Citation: X Raurell., et al. "Intraventricular Tension Pneumocephalus and Pneumorrhachis in a Dog; Diagnosis, Treatment, and MRI Follow-Up". Acta Scientific Veterinary Sciences 2.3 (2020): 57-60. 